\title{
L-Asparaginase-Induced Pancreatic Injury is Associated with an Imbalance in Plasma Amino Acid Levels
}

Kei Minowa, ${ }^{1}$ Mitsuyoshi Suzuki, ${ }^{1}$ Junya Fujimura, ${ }^{1}$ Masahiro Saito, ${ }^{1}$ Katsuyoshi Koh ${ }^{2}$ Akira Kikuchi, ${ }^{3}$ Ryoji Hanada ${ }^{2}$ and Toshiaki Shimizu ${ }^{1}$

1 Department of Pediatrics, Juntendo University School of Medicine, Tokyo, Japan

2 Division of Hematology and Oncology, Saitama Children's Medical Center, Saitama, Japan

3 Department of Pediatrics, Teikyo University, Tokyo, Japan

\section{Abstract}

Background: The use of L-asparaginase (ASNase) to modify amino acid metabolism is one of the most effective chemotherapeutic means of inducing remission in acute lymphoblastic leukemia (ALL). However, severe pancreatitis sometimes occurs in patients receiving ASNase, because of an unknown mechanism.

Objective: The purpose of the present study was to evaluate the relationship between ASNase-induced pancreatic injury and plasma amino acid levels in patients undergoing ASNase therapy.

Methods: A total of 29 children aged 1-13.25 years (median age 4 years; male: female ratio 19:10) with ALL, who received induction therapy according to the Tokyo Children's Cancer Study Group L04-16 protocol, were studied. Levels of plasma amino acids and serum rapid turnover proteins (RTPs), pancreatic enzymes, and pancreatic protease inhibitors were measured before and 1, 2, 3, 4, 5, and 7 weeks after the first administration of ASNase.

Results: Plasma asparagine levels were significantly lower after the first injection of ASNase $(\mathrm{p}<0.01)$ and had almost recovered 2 weeks after the last ASNase injection. At 4 weeks after the first ASNase injection, serum aspartic acid, trypsin, and pancreatic secretory trypsin inhibitor (PSTI) levels remained significantly higher than those before the first ASNase injection $(\mathrm{p}<0.01)$, and serum levels of prealbumin and transferrin remained significantly lower than those before the first ASNase injection $(\mathrm{p}<0.01)$. Plasma amino acid and serum RTP levels gradually normalized after the last ASNase injection.

Conclusions: Levels of serum trypsin and PSTI were elevated during the 2 weeks after administration of ASNase, which suggested the presence of subclinical pancreatitis. This period is similar to the time period in the present 
study when the levels of plasma amino acids changed, thus suggesting that ASNase-induced pancreatic injury could be caused by the imbalance of plasma amino acid levels.

\section{Introduction}

L-asparaginase (ASNase) is an important oncotherapy, particularly for acute lymphoblastic leukemia (ALL). ASNase is thought to exert its anti-tumor activity by hydrolyzing asparagine to aspartate and ammonia. Asparagine synthetase activity in some malignant lymphoblasts is very low, and the lymphoblasts rely on an exogenous supply of amino acids. Lymphoblasts thereby deplete the supply of asparagine, which leads to cell death. ${ }^{[1-3]}$ An advantage of ASNase is that it does not have cross-resistance with other anti-tumor agents. Another important advantage is that it has low toxicity in normal tissues and in other neoplastic cells that express high levels of asparagine synthetase. Potential side effects of ASNase include hypersensitivity reactions, central nervous system dysfunction, coagulation abnormalities, liver dysfunction, hyperglycemia, hyperlipemia, and pancreatitis. ${ }^{[4]}$

In some cases, ASNase-induced pancreatitis can be life threatening and all chemotherapy must be discontinued. Although patients can recover from this kind of acute pancreatitis, re-initiation of therapy with ASNase in such cases is generally considered contraindicated. There are various treatments for ASNase-induced pancreatitis: some reports have suggested use of octreotide ${ }^{[5-7]}$ or a continuous regional arterial infusion of a protease inhibitor and antibiotics. ${ }^{[8]}$ Although pancreatitis remains one of the most severe complications of ASNase therapy, it is impossible to predict who will develop pancreatic toxicity from ASNase. ${ }^{[9]}$ Furthermore, there is no adequate prophylaxis for this potentially life-threatening condition.

In the present study, the pharmacologic effects of ASNase on plasma amino acid levels and serum rapid turnover protein (RTP) levels were investigated as factors potentially related to ASNase-induced pancreatic injury. The presence of pancreatitis or pancreatic injury during administration of ASNase was evaluated through measurement of the levels of serum pancreatic enzymes and pancreatic protease inhibitors.

\section{Methods}

\section{Subjects}

The study group consisted of 29 children aged 1 year to 13.25 years (median age 4 years; male: female ratio $19: 10$ ) who were newly diagnosed with ALL and received chemotherapy for ALL (B-cell phenotype: T-cell phenotype ratio $25: 4$ ). Patients were classified as standard risk (SR) if they met the following criteria: age 1-9.99 years and white blood cell count $<50000 / \mu \mathrm{L}$. All others were designated as high risk (HR) [SR : HR ratio $18: 11]$. Induction therapy was initiated according to the Tokyo Children's Cancer Study Group L04-16 protocol and consisted of prednisolone $60 \mathrm{mg} / \mathrm{m}^{2} /$ day (on days $1-35$, tapering off on days 36-42); vincristine $1.5 \mathrm{mg} / \mathrm{m}^{2} /$ day (on days 8,15 , 22,29 , and 36); daunomycin $25 \mathrm{mg} / \mathrm{m}^{2} /$ day (on days 10, 11, 31, and 32); ASNase derived from Escherichia coli (Leunase; Kyowa Hakko Kirin, Tokyo, Japan) $6000 \mathrm{IU} / \mathrm{m}^{2} /$ day (on days 15,17 , 19, 22, 24, 26, 29, 31, and 33); cyclophosphamide $1 \mathrm{~g} / \mathrm{m}^{2} /$ day (on days 9 and 30); and intrathecal administration of hydrocortisone sodium phosphate $25 \mathrm{mg}$ /day, methotrexate $12.5 \mathrm{mg} /$ day, and cytarabine $25 \mathrm{mg}$ /day (on days 8 and 22) [figure 1]. The study protocol was approved by the ethics committee of the Juntendo University School of Medicine. Informed consent was obtained from all patients or their parents before participation in the study.

\section{Samples}

Blood samples were collected before the first injection of ASNase (day 15) and at 1 week (day 22), 2 weeks (day 29), 3 weeks (day 36), 4 weeks 


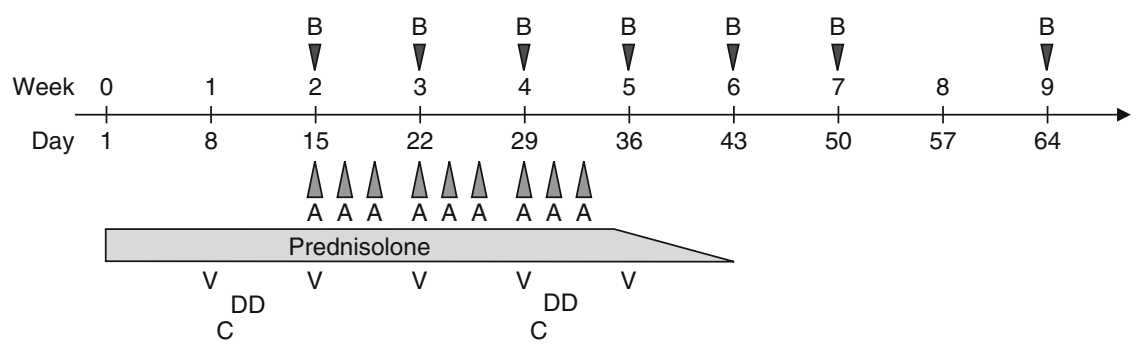

Fig. 1. Induction therapy regimen of the Tokyo Children's Cancer Study Group L04-16 protocol. Blood samples were collected on days 15,22 , $29,36,43,50$, and 64 . Patients received L-asparaginase $6000 \mathrm{IU} / \mathrm{m}^{2} /$ day on days $15,17,19,22,24,26,29,31$, and 33 . Patients received prednisolone $60 \mathrm{mg} / \mathrm{m}^{2} /$ day on days $1-35$, tapering off on days $36-42$. Patients received vincristine $1.5 \mathrm{mg} / \mathrm{m}^{2} /$ day on days $8,15,22,29$, and 36. Patients received daunomycin $25 \mathrm{mg} / \mathrm{m}^{2} /$ day on days $10,11,31$, and 32 . Patients received cyclophosphamide $1 \mathrm{~g} / \mathrm{m}^{2} /$ day on days 9 and 30. $\mathbf{A}=\mathrm{L}$-asparaginase; $\mathbf{B}=$ blood; $\mathbf{C}=$ cyclophosphamide; $\mathbf{D}=$ daunomycin; $\mathbf{V}=$ vincristine.

(day 43), 5 weeks (day 50), and 7 weeks (day 64) after the first injection of ASNase. Blood samples were used for measurement of levels of serum amylase, lipase, trypsin, pancreatic protease inhibitors (pancreatic secretory trypsin inhibitor [PSTI], $\alpha 1$-antitrypsin [ $\alpha 1$-AT], and $\alpha 2$-macroglobulin $[\alpha 2-\mathrm{M}]$ ), and RTPs (prealbumin [PA], transferrin [Tf], and retinol-binding protein $[\mathrm{RBP}])$, and plasma amino acids. In the present study, serum levels of RTPs were investigated as products that are induced by metabolism of plasma amino acids. After day 33, all patients continued to receive other oncolytic agents but did not receive ASNase during induction therapy.

\section{Assays}

Blood samples were divided into two groups. One group was placed in heparinized tubes (Nipro Co., Ltd., Tokyo, Japan) and immediately centrifuged at $3000 \mathrm{rpm}$ for 5 minutes at $-4^{\circ} \mathrm{C}$. Plasma was mixed with an equal volume of $10 \%$ sulfosalicylic acid (w/v) under ice for rapid deproteinization and inactivation of ASNase. ${ }^{[10]}$ The mixture was centrifuged, and the supernatant was used as the sample solution. Amino acid analysis was performed with high-performance liquid chromatography after precolumn derivation with o-phthaldialdehyde, as previously described, using an L-8500 Amino Acid Analyzer (Hitachi Co., Ltd., Tokyo, Japan).$^{[11]}$ Plasma amino acid levels are expressed in nanomoles per milliliter $(\mathrm{nmol} / \mathrm{mL})$. Plasma amino acid levels were measured twice to ensure accuracy.
The second group of blood samples was collected in tubes containing a serum separating agent and coagulation promotion film (Nipro Co., Ltd., Osaka, Japan), and separation was performed by centrifugation at $3000 \mathrm{rpm}$ for $10 \mathrm{~min}$ utes at $22^{\circ} \mathrm{C}$. Serum levels of amylase and lipase were measured in our institutional laboratory, the accuracy of which was assured by the Japanese Society of Laboratory Medicine. Results are expressed in international units per liter (IU/L). Trypsin was measured by a radioimmunoassay (RIA-Gnost Trypsin II Kit; Nihon Schering Co., Ltd., Osaka, Japan). PSTI was measured by a radioimmunoassay (Ab-Bead PSTI Kit; Eiken Chemical Co., Ltd., Tokyo, Japan). Trypsin and PSTI levels are expressed in nanograms per milliliter $(\mathrm{ng} / \mathrm{mL})$. The levels of $\alpha 1-\mathrm{AT}$ and $\alpha 2-\mathrm{M}$ were determined by the nephelometry method with a BN II Analyzer (Dade Behring GmbH, Marburg, Germany). The results of both protein measurements are expressed in milligrams per deciliter $(\mathrm{mg} / \mathrm{dL})$. The levels of PA and RBP were measured by the nephelometry method with a BN II Analyzer (Dade Behring Co., Ltd., Tokyo, Japan). Serum Tf levels were determined on a JCA-BM12 Biochemical Analyzer (Japan Electron Optics Laboratory Co., Ltd., Tokyo, Japan) with a turbidimetric immunoassay (N-Assay TIA Tf-H Nittobo; Nitto Boseki Co., Ltd., Tokyo, Japan). The RTP levels are expressed in milligrams per deciliter $(\mathrm{mg} / \mathrm{dL})$. Serum pancreatic enzyme, pancreatic protease inhibitor, and RTP levels were measured twice to ensure accuracy. 


\section{Statistics}

Values are presented as the mean \pm standard deviation (SD). Statistical analysis was performed with the non-parametric Friedman test. SPSS statistical analysis software (IBM SPSS Statistics Version 19) was used for all computations. A p-value of $<0.05$ was considered statistically significant.

\section{Results}

One patient (a 1-year-old girl) developed ASNase-induced pancreatitis. The results for the rest of the cases $(n=28)$ were as follows.

\section{Plasma Amino Acid Levels}

Plasma asparagine levels after the first injection of ASNase were significantly lower than those before the ASNase injection $(\mathrm{p}<0.01)$. Plasma asparagine reached minimum levels 2 weeks after the first injection, gradually increased, and had almost recovered at 5 weeks after the first injection. Serum aspartic acid levels at 1, 2, 3, and 4 weeks after the first ASNase injection were significantly higher than those before the ASNase injection $(p<0.01)$. Levels of most of the other amino acids fluctuated 1, 2, and 3 weeks after the first injection, and there were almost no differences between the levels before the first ASNase injection and those 5 and 7 weeks after the first injection (table I).

\section{Serum Rapid Turnover Protein Levels}

Serum levels of RTPs rapidly decreased after the first ASNase injection. Serum levels of PA and $\mathrm{Tf}$ at 1, 2, 3, and 4 weeks after the first ASNase injection were significantly lower than those before the first ASNase injection $(p<0.01)$. Serum levels of RTPs reached minimum levels 2 weeks after the first ASNase injection and then gradually increased (table II).

\section{Serum Pancreatic Enzyme and Pancreatic Protease Inhibitor Levels}

Serum levels of amylase and lipase did not fluctuate during the observation period. Serum trypsin levels at 2, 3, and 4 weeks after the first ASNase injection were significantly higher than those before the first ASNase injection $(\mathrm{p}<0.01)$. Serum PSTI levels at 2, 3, and 4 weeks after the first ASNase injection were also higher than those before the first ASNase injection $(\mathrm{p}<0.01)$. Serum levels of $\alpha 1-A T$ and $\alpha 2-M$ remained unchanged during ASNase therapy (table II).

\section{The Patient Who Developed Pancreatitis}

A 15-month-old girl who developed pancreatitis experienced nausea and upper abdominal pain on the day after the fourth ASNase injection (day 22).

Table I. Time course of plasma amino acid levels

\begin{tabular}{|c|c|c|c|c|c|c|c|}
\hline \multirow[t]{2}{*}{ Amino acid } & \multirow[b]{2}{*}{$\begin{array}{l}\text { Level before } \\
\text { ASNase } \\
\text { injection } \\
\text { [days 13-15] } \\
(\mathrm{nmol} / \mathrm{mL})\end{array}$} & \multicolumn{6}{|c|}{ Level after ASNase injection (nmol/L) } \\
\hline & & $\begin{array}{l}\text { Week } 1 \\
\text { [day 22] }\end{array}$ & $\begin{array}{l}\text { Week } 2 \\
\text { [day 29] }\end{array}$ & $\begin{array}{l}\text { Week } 3 \\
\text { [day 36] }\end{array}$ & $\begin{array}{l}\text { Week } 4 \\
\text { [days 41-43] }\end{array}$ & $\begin{array}{l}\text { Week } 5 \\
\text { [days 48-50] }\end{array}$ & $\begin{array}{l}\text { Week } 7 \\
\text { [days 64-66] }\end{array}$ \\
\hline Aspartic acid & $2.7 \pm 2.2$ & $8.4 \pm 4.7^{* *}$ & $9.9 \pm 6.4^{* *}$ & $12.6 \pm 9.5^{* *}$ & $13.2 \pm 8.4^{* *}$ & $6.1 \pm 5.9$ & $3.2 \pm 3$ \\
\hline Asparagine & $46.2 \pm 13.9$ & $2.3 \pm 8.0^{* * *}$ & $0 \pm 0^{* * *}$ & $2.6 \pm 7.7^{* *}$ & $7.5 \pm 15.3^{* * *}$ & $36.7 \pm 14.3^{* *}$ & $37.1 \pm 8.6^{* *}$ \\
\hline Glutamic acid & $58.4 \pm 20.1$ & $160.5 \pm 123.7^{* * *}$ & $160.4 \pm 109.1^{* * *}$ & $190.1 \pm 148.5^{* * *}$ & $90.3 \pm 46.0$ & $69.0 \pm 23.3$ & $50.0 \pm 14.0$ \\
\hline Glutamine & $453.8 \pm 89.3$ & $294.3 \pm 124.7^{* * *}$ & $295.9 \pm 145.6^{* * *}$ & $294.9 \pm 162.8^{* * *}$ & $380.2 \pm 103.2^{* * *}$ & $462.8 \pm 107.9$ & $464.6 \pm 82.6$ \\
\hline Threonine & $124.6 \pm 44.3$ & $148.4 \pm 96.8$ & $216.9 \pm 96.8^{* *}$ & $221.4 \pm 110.0^{* *}$ & $136.4 \pm 69.2$ & $106.9 \pm 46.6$ & $103.8 \pm 33.0$ \\
\hline Cystine & $37.2 \pm 10.0$ & $33.5 \pm 8.0$ & $32.9 \pm 8.7$ & $32.2 \pm 10.5^{*}$ & $37.1 \pm 9.5$ & $42.3 \pm 9.4$ & $40.8 \pm 8.1^{*}$ \\
\hline Methionine & $22.4 \pm 7.8$ & $20.3 \pm 6.7$ & $22.9 \pm 8.3$ & $24.3 \pm 9.2$ & $18.1 \pm 7.5$ & $20.6 \pm 6.9$ & $19.0 \pm 5.4$ \\
\hline Lysine & $189.1 \pm 58.1$ & $137.1 \pm 42.3^{* * *}$ & $138.5 \pm 39.1^{* *}$ & $168.7 \pm 76.5$ & $156.7 \pm 57.5^{*}$ & $160.7 \pm 52.3^{*}$ & $154.3 \pm 42.2^{*}$ \\
\hline Arginine & $75.4 \pm 34.7$ & $48.8 \pm 21.9^{* *}$ & $51.4 \pm 21.4^{* *}$ & $63.3 \pm 30.0^{*}$ & $57.1 \pm 23.4^{* *}$ & $60.7 \pm 33.5^{*}$ & $56.2 \pm 22.7^{* *}$ \\
\hline Citrulline & $17.6 \pm 7.0$ & $18.2 \pm 6.8$ & $19.9 \pm 8.7$ & $19.4 \pm 9.0$ & $17.8 \pm 7.7$ & $20.2 \pm 4.8$ & $16.5 \pm 6.3$ \\
\hline
\end{tabular}


Table II. Time course of serum pancreatic enzyme, pancreatic protease inhibitor, and rapid turnover protein levels

\begin{tabular}{|c|c|c|c|c|c|c|c|}
\hline \multirow[t]{2}{*}{ Parameter } & \multirow{2}{*}{$\begin{array}{l}\text { Level before } \\
\text { ASNase } \\
\text { injection } \\
\text { [days 13-15] }\end{array}$} & \multicolumn{6}{|c|}{ Level after ASNase injection } \\
\hline & & $\begin{array}{l}\text { Week } 1 \\
\text { [day 22] }\end{array}$ & $\begin{array}{l}\text { Week } 2 \\
\text { [day 29] }\end{array}$ & $\begin{array}{l}\text { Week } 3 \\
\text { [day 36] }\end{array}$ & $\begin{array}{l}\text { Week } 4 \\
\text { [days 41-43] }\end{array}$ & $\begin{array}{l}\text { Week } 5 \\
\text { [days 48-50] }\end{array}$ & $\begin{array}{l}\text { Week } 7 \\
\text { [days 64-66] }\end{array}$ \\
\hline Amylase (IU/L) & $60.6 \pm 27.6$ & $56.0 \pm 28.5$ & $55.3 \pm 25.4$ & $52.1 \pm 21.3$ & $51.4 \pm 22.5$ & $55.2 \pm 22.2$ & $55.7 \pm 39.1$ \\
\hline Lipase (IU/L) & $18.7 \pm 8.2$ & $16.2 \pm 4.6$ & $19.1 \pm 8.9$ & $20.7 \pm 9.0$ & $21.3 \pm 6.4$ & $23.8 \pm 8.1$ & $29.2 \pm 35.7$ \\
\hline Trypsin (ng/mL) & $285.7 \pm 142.3$ & $326.5 \pm 131.2$ & $330 \pm 133.5^{* *}$ & $371.3 \pm 157.5^{* * *}$ & $362.2 \pm 202.9^{* *}$ & $306.4 \pm 170.2$ & $324.1 \pm 470.4$ \\
\hline \multicolumn{8}{|c|}{ Pancreatic protease inhibitors } \\
\hline PSTI (ng/mL) & $10.7 \pm 4.9$ & $35.7 \pm 24.8$ & $83.1 \pm 83.6^{* *}$ & $74.9 \pm 47.8^{* *}$ & $53.3 \pm 48.3^{* *}$ & $12.2 \pm 7.0$ & $13.2 \pm 10.0$ \\
\hline$\alpha 1-\mathrm{AT}(\mathrm{mg} / \mathrm{dL})$ & $139.3 \pm 43.3$ & $120 \pm 33.8$ & $113.5 \pm 31.5$ & $131.6 \pm 48.6$ & $168.6 \pm 50.2^{* *}$ & $145.6 \pm 54.8$ & $148.7 \pm 40.5$ \\
\hline$\alpha 2-\mathrm{M}(\mathrm{mg} / \mathrm{dL})$ & $271.1 \pm 45.4$ & $263.0 \pm 51.8$ & $250.3 \pm 42.7$ & $265.5 \pm 55.8$ & $302.1 \pm 69.8$ & $354.0 \pm 60.4^{* *}$ & $346.5 \pm 75.7^{* *}$ \\
\hline \multicolumn{8}{|c|}{ Rapid turnover proteins } \\
\hline $\mathrm{PA}(\mathrm{mg} / \mathrm{dL})$ & $33.8 \pm 7.8$ & $23.0 \pm 7.0^{* *}$ & $18.7 \pm 7.2^{* * *}$ & $25.3 \pm 11.4^{* *}$ & $26.8 \pm 11.4^{* *}$ & $22.3 \pm 7.0^{* * *}$ & $23.5 \pm 8.1^{* *}$ \\
\hline $\mathrm{RBP}(\mathrm{mg} / \mathrm{dL})$ & $4.3 \pm 1.0$ & $3.1 \pm 1.2^{* *}$ & $2.6 \pm 1.0^{* * *}$ & $4.3 \pm 4.4^{* *}$ & $4.2 \pm 2.0$ & $3.2 \pm 0.9^{* *}$ & $3.4 \pm 1.3^{* *}$ \\
\hline $\mathrm{Tf}(\mathrm{mg} / \mathrm{dL})$ & $233.3 \pm 46.4$ & $164.9 \pm 40.3^{* *}$ & $120.7 \pm 34.5^{* *}$ & $120.7 \pm 41.2^{* *}$ & $154.2 \pm 50.0^{* *}$ & $208.1 \pm 30.8^{* *}$ & $172.6 \pm 24.1^{* * *}$ \\
\hline
\end{tabular}

She was diagnosed as having ASNase-induced pancreatitis by elevated levels of serum pancreatic enzymes and findings of abdominal computed tomography. Her serum PSTI level was also higher than that before the first ASNase injection, and her serum levels of $\alpha 1-\mathrm{AT}$ and $\alpha 2-\mathrm{M}$ remained unchanged on that day (day 22). Changes in her serum amino acid levels between day 15 and day 22 were similar to the results in patients who did not develop acute pancreatitis. Though she recovered from the pancreatitis after 2 weeks of conservative therapy, it was deemed unsafe to use ASNase with the rest of her oncotherapy, for fear of recurrent pancreatitis.

\section{Discussion}

Because of use of other chemotherapeutic agents (including steroids) during oncotherapy, the mechanisms of ASNase-induced pancreatitis in humans remain unknown. Although there have been many reports of ASNase-induced pancreatitis, ${ }^{[6,9,12-16]}$ few studies have examined the relationship between ASNase therapy and acute pancreatitis by measuring changes in serum levels of pancreatic enzymes or plasma levels of amino acids. ${ }^{[15,17,18]}$ As in previous studies, ${ }^{[19,20]}$ in the present study the plasma asparagine levels decreased rapidly after the first ASNase injection.
On the other hand, the levels of plasma aspartic acid increased. By 4 weeks after the first injection of ASNase, these changes had gradually normalized, and almost normal levels of asparagine and aspartic acid were seen 5 weeks after the first injection of ASNase. Levels of other amino acids changed during the first week after the injection of ASNase and recovered 4 weeks after the first injection of ASNase. These results suggest that it takes about 2 weeks for the imbalance of plasma amino acid levels after the last injection of ASNase to improve.

RTP levels in the serum rapidly decreased after the first ASNase injection and gradually normalized during the 4 weeks after the first injection. These changes suggest that the imbalance of plasma amino acids prevents intracellular utilization of amino acids, and a decrease in RTP levels could be a result of this imbalance. Not only administration of ASNase during chemotherapy but also other therapeutic drugs and anorexia have been implicated as factors capable of inducing these changes. However, the present results indicate that levels of plasma amino acids and serum RTPs gradually normalize after the last ASNase injection, despite continued use of other chemotherapeutic drugs.

Serum amylase and lipase levels were unchanged during the present study, though serum 
trypsin levels increased after the ASNase injection. Serum PSTI levels increased after the ASNase injection as well. Acute pancreatitis develops with unregulated trypsin activity after breakdown of critical protective mechanisms and copious secretion of pancreatic enzymes such as amylase and lipase. ${ }^{[21,22]}$ The present results indicate that inhibitors of trypsin could potentially prevent development of pancreatitis, and suggest the presence of subclinical pancreatitis in cases who do not develop pancreatitis during administration of ASNase. Not only ASNase but also prednisolone has been implicated as an agent capable of inducing pancreatitis. ${ }^{[23]}$ Previous reports suggest that ASNase is the more likely source of pancreatitis on the basis of histologic examination of the pancreas, the relative infrequency of prednisolone-induced pancreatitis, and a negative result after rechallenge with prednisolone. ${ }^{[14,18,24]}$

In the present study, one of 29 patients $(3 \%)$ developed ASNase-induced pancreatitis, similar to the morbidity rates in previous reports. ${ }^{[4,6,9,16,25]}$ Since the patient developed severe pancreatitis, ASNase was contraindicated during the rest of her treatment for ALL. The results of her blood tests were similar to the results from those patients who did not develop acute pancreatitis, so there was no parameter that could be used to predict acute pancreatitis. When ASNase-induced pancreatitis occurs, treatment with Erwinia chrysanthemi asparaginase is an option. As it can also lead to pancreatitis, Erwinia asparaginase is a second-line therapy for ALL after hypersensitivity to Escherichia coli asparaginase. ${ }^{[26]}$ Furthermore, there are no widely accepted guidelines for use of Erwinia asparaginase, and such treatment is not covered by health insurance providers in Japan. Previous reports have shown that there is a mean of almost 10 days from the last administration of ASNase to diagnosis of pancreatitis. ${ }^{[5,9,16]}$ Similarly, Japanese case reports of ASNaseinduced pancreatitis have shown that 50 of 56 patients $(89 \%)$ who developed ASNase-induced pancreatitis did so within 10 days (median 2 days, range 0-23 days) after administration of ASNase. ${ }^{[27]}$ This period is similar to the time period in the present study when the levels of plasma amino acids, serum trypsin, and serum PSTI changed.
In the rat model, it has been proposed that ASNase-induced pancreatic injury can involve disruption of the plasma amino acid balance that is caused by ASNase. Disruption of protein synthesis in acinar cells then causes inhibition of exocytosis following the histologic morphologic changes. ${ }^{[28]}$ The present results imply that the plasma amino acid level imbalance could also be a factor in ASNaseinduced pancreatitis in humans. In clinical cases, there would also be other triggers of pancreatitis in addition to the plasma amino acid level imbalance.

Though there is no well established prophylaxis for ASNase-induced pancreatic injury, it has been reported that an ALL patient was successfully retreated using ASNase with octreotide after an episode of ASNase-induced pancreatitis. ${ }^{[29,30]}$ Octreotide is capable of inhibiting pancreatic uptake of plasma amino acids, and this inhibition could be an important mechanism by which octreotide decreases pancreatic enzyme secretion. ${ }^{[31]}$ It is thought that octreotide could prevent ASNaseinduced pancreatic injury through its physiopathologic properties. Recently, Muwakkit et al. have also suggested that allopurinol, which is an inhibitor of xanthine oxidase, has a preventive effect on ASNase-induced pancreatitis. ${ }^{[32]}$

\section{Conclusion}

An imbalance of plasma amino acid levels during the 2 weeks after administration of ASNase was observed. In this period, elevations of serum trypsin and PSTI levels were also observed, indicating the possible presence of subclinical pancreatitis in the patients who did not develop pancreatitis. This imbalance of plasma amino acid levels normalized after ASNase was discontinued, even though other chemotherapy for ALL continued. This plasma amino acid imbalance could be one factor behind ASNase-induced pancreatitis and pancreatic injury in humans. Further research should focus on prophylaxis for ASNase-induced pancreatic injury, which could greatly improve treatment outcomes of ALL in children.

\section{Acknowledgments}

This study was supported in part by a grant from the Ministry of Education, Culture, Sports, Science, and Technology 
of Japan (grant no. 21791010). The authors have no conflicts of interest that are directly relevant to the contents of this study.

\section{References}

1. Richards NG, Kilberg MS. Asparagine synthetase chemotherapy. Annu Rev Biochem 2006; 75: 629-54

2. Avramis VI, Panosyan EH. Pharmacokinetic/pharmacodynamic relationships of asparaginase formulations: the past, the present and recommendations for the future. Clin Pharmacokinet 2005; 44: 367-93

3. Ohnuma T, Holland JF, Freeman A, et al. Biochemical and pharmacological studies with asparaginase in man. Cancer Res 1970; 30: 2297-305

4. Muller HJ, Boos J. Use of L-asparaginase in childhood ALL. Crit Rev Oncol Hematol 1998; 28: 97-113

5. Wu SF, Chen AC, Peng CT, et al. Octreotide therapy in asparaginase-associated pancreatitis in childhood acute lymphoblastic leukemia. Pediatr Blood Cancer 2008; 51: 824-5

6. Sahu S, Saika S, Pai SK, et al. L-asparaginase (Leunase) induced pancreatitis in childhood acute lymphoblastic leukemia. Pediatr Hematol Oncol 1998; 15: 533-8

7. Garrington T, Bensard D, Ingram JD, et al. Successful management with octreotide of a child with L-asparaginase induced hemorrhagic pancreatitis. Med Pediatr Oncol 1998; 30: 106-9

8. Morimoto A, Imamura $\mathrm{T}$, Ishii $\mathrm{R}$, et al. Successful management of severe L-asparaginase-associated pancreatitis by continuous regional arterial infusion of protease inhibitor and antibiotic. Cancer 2008; 113: 1362-9

9. Knoderer HM, Robarge J, Flockhart DA. Predicting asparaginase-associated pancreatitis. Pediatr Blood Cancer 2007; 49: 634-9

10. Gentili D, Zucchetti M, Conter V, et al. Determination of L-asparagine in biological samples in the presence of L-asparaginase. J Chromatogr B Biomed Appl 1994; 657: 47-52

11. Deyl Z, Hyanek J, Horakova M. Profiling of amino acids in body fluids and tissues by means of liquid chromatography. J Chromatogr 1986; 379: 177-250

12. Cairo MS. Adverse reactions of L-asparaginase. Am J Pediatr Hematol Oncol 1982; 4: 335-9

13. Weetman RM, Baehner RL. Latent onset of clinical pancreatitis in children receiving L-asparaginase therapy. Cancer 1974; 34: 780-5

14. Land VJ, Sutow WW, Fernbach DJ, et al. Toxicity of L-asparginase in children with advanced leukemia. Cancer 1972; 30: 339-47

15. Haskell CM, Canellos GP, Leventhal BG, et al. Lasparaginase: therapeutic and toxic effects in patients with neoplastic disease. N Engl J Med 1969; 281: 1028-34

16. Alvarez OA, Zimmerman G. Pegaspargase-induced pancreatitis. Med Pediatr Oncol 2000; 34: 200-5

17. Shimizu T, Yamashiro Y, Igarashi J, et al. Increased serum trypsin and elastase-1 levels in patients undergoing L-asparaginase therapy. Eur J Pediatr 1998; 157: 561-3
18. Greenstein R, Nogeire C, Ohnuma T, et al. Management of asparaginase induced hemorrhagic pancreatitis complicated by pseudocyst. Cancer 1979; 43: 718-22

19. Avramis VI, Sencer S, Periclou AP, et al. A randomized comparison of native Escherichia coli asparaginase and polyethylene glycol conjugated asparaginase for treatment of children with newly diagnosed standard-risk acute lymphoblastic leukemia: a Children's Cancer Group study. Blood 2002; 99: 1986-94

20. Boos J, Werber G, Ahlke E, et al. Monitoring of asparaginase activity and asparagine levels in children on different asparaginase preparations. Eur J Cancer 1996; 32: 1544-50

21. Elfar M, Gaber LW, Sabek O, et al. The inflammatory cascade in acute pancreatitis: relevance to clinical disease. Surg Clin North Am 2007; 87: 1325-40

22. Whitcomb DC. Value of genetic testing in the management of pancreatitis. Gut 2004; 53: 1710-7

23. Mallory A, Kern F. Drug-induced pancreatitis. Bailliere's Clin Gastroenterol 1988; 2: 293-307

24. McArthur KE. Drug-induced pancreatitis. Aliment Pharmacol Ther 1996; 10: 23-38

25. McLean R, Martin S, Lam-Po-Tang PR. Fatal case of L-asparaginase induced pancreatitis. Lancet 1982; 2: $1401-2$

26. Vrooman LM, Supko JG, Neuberg DS, et al. Erwinia asparaginase after allergy to E. coli asparaginase in children with acute lymphoblastic leukemia. Pediatr Blood Cancer 2010; 54: 199-205

27. Minowa K, Suzuki M, Naritaka N, et al. Clinical course and outcome of L-asparaginase-induced pancreatitis in children [abstract]. J Jpn Pediatr Soc 2011; 115: 410

28. Suzuki M, Shimizu T, Kudo T, et al. Octreotide prevents L-asparaginase-induced pancreatic injury in rats. Exp Hematol 2008; 36: 172-80

29. Suzuki M, Takata O, Sakaguchi S, et al. Retherapy using $\mathrm{L}$-asparaginase with octreotide in a patient recovering from L-asparaginase-induced pancreatitis. Exp Hematol 2008; 36: $253-4$

30. Tokimasa S, Yamato K. Does octreotide prevent Lasparaginase-associated pancreatitis in children with acute lymphoblastic leukaemia? Br J Haematol 2012; 157 (3): $381-2$

31. Gullo L, Pezzilli R, Ancona D, et al. Effect of octreotide, a long-acting somatostatin analogue, on plasma amino acid uptake by the pancreas. Pancreas 1991; 6: 668-72

32. Muwakkit S, Saab R, Yazbeck N, et al. L-asparaginase induced pancreatitis in children with acute lymphoblastic leukemia: is allopurinol protective? Pediatr Hematol Oncol 2010; 27: 496-501

Correspondence: Dr Kei Minowa, Department of Pediatrics, Juntendo University School of Medicine, 2-1-1 Hongo, Bunkyo-ku, Tokyo 113 8421, Japan.

E-mail: kei.miowakei@gmail.com 